\title{
Ensino de saúde do trabalhador nos cursos de graduação em Terapia Ocupacional: contribuições ao debate sobre parâmetros de avaliação do ensino público brasileiro*
}

\section{Education of worker's health in Occupational Therapy undergraduate courses: contributions to the discussion on evaluation parameters of Brazilian public education}

\author{
Iranise Moro Pereira Jorge ${ }^{1}$, Angela Paula Simonelli ${ }^{2}$, Jessica Elisa da Rosa ${ }^{3}$, \\ Sattylla Sanny de Matos Ferreira ${ }^{4}$, Marina Batista Chaves Azevedo de Souza ${ }^{5}$, \\ Barbara Iansã de Lima Barroso ${ }^{6}$
}

http://dx.doi.org/10.11606/issn.2238-6149.v27i2p109-115

Pereira Jorge IM, Simonelli AP, Rosa JE, Ferreira SSM, Souza MBCA, Barroso BIL. Ensino de saúde do trabalhador nos cursos de graduação em Terapia Ocupacional: contribuições ao debate sobre parâmetros de avaliação do ensino público brasileiro. Rev Ter Ocup Univ Sao Paulo. 2016 maio-ago.;27(2):109-15.

RESUMO: Esta pesquisa possui como objetivo, investigar as ações de pesquisa, ensino e extensão desenvolvidas na área de Saúde do Trabalhador nos cursos de graduação em Terapia Ocupacional das universidades públicas do Brasil. Trata-se de pesquisa descritiva exploratória, realizada por duas Universidades Federais. A amostra foi composta por $(n=16)$ cursos de graduação em Terapia Ocupacional de $(n=15)$ universidades públicas. Os dados encontrados demonstram que as universidades abordam a área de Saúde do Trabalhador. Dispõem, em sua maioria, de professores com titulação na área de Ergonomia e apresentam, nos últimos três anos, $(\mathrm{n}=08)$ campos de estágio, $(n=14)$ projetos de extensão, $(n=70)$ trabalhos de conclusão de curso, $(n=20)$ projetos de pesquisas e $(n=29)$ publicações em periódicos científicos. Destaca-se a necessidade de ações que promovam a intersetorialização e o debate entre instituições, a fim de possibilitar troca de experiências e avanços no conteúdo do ensino, tanto neste campo de atuação, quanto na formação dos docentes responsáveis pela área de Saúde do Trabalhador.

DESCRITORES: Educação superior; Terapia ocupacional/ educação; Saúde do trabalhador/educação.
Pereira Jorge IM, Simonelli AP, Rosa JE, Ferreira SSM, Souza MBCA, Barroso BIL. Education of worker's health in Occupational Therapy undergraduate courses: contributions to the discussion on evaluation parameters of Brazilian public education. Rev Ter Ocup Univ Sao Paulo. 2016 May-Aug.;27(2):109-15.

\begin{abstract}
This study aims to investigate actions of research, education and extension developed on worker's health field in undergraduate courses of Occupational Therapy in public universities in Brazil. It is a descriptive and exploratory research, conducted by two federal universities. The sample was formed by $(n=16)$ Occupational Therapy undergraduate courses $(n=15)$ in public universities. The data obtained demonstrated that the universities approach worker's health field. They have, in most cases, professors with titration in ergonomics and, in the last three years, $(n=08)$ internship fields, $(n=14)$ extension projects, $(n=70)$ and a final work for undergraduates, $(n=20)$ projects and researches, and $(n=29)$ publications in scientific journals. Actions to promote sector union and discussions between institutions are needed, to enable experience exchanges and progress in education matter, both in this field and formation of teachers responsible for worker's health area.
\end{abstract}

KEYWORDS: Education higher; Occupational therapy/ education; Occupational health/education.

*Artigo inédito elaborado a partir de dados coletados durante consórcio de pesquisa entre os Laboratório de Reabilitação, Acessibilidade e Trabalho (LABRAT) da Universidade Federal do Paraná e o Laboratório de Saúde, Trabalho e Ergonomia (LASTE) da Universidade Federal da Paraíba, sendo parte da pesquisa de Iniciação Científica das alunas Jessica Elisa da Rosa e Sattylla Sanny de Matos Ferreira, desenvolvida na Universidade Federal do Paraná. Todas as autoras participaram efetivamente das etapas de produção deste artigo.

1. Professora Adjunta do Departamento de Terapia Ocupacional, Laboratório de Reabilitação, Acessibilidade e Trabalho (LABRAT).

2. Professora Adjunta do Departamento de Terapia Ocupacional, Laboratório de Reabilitação, Acessibilidade e Trabalho (LABRAT).

3. Acadêmica do Curso de Terapeuta Ocupacional. Laboratório de Reabilitação, Acessibilidade e Trabalho (LABRAT).

4. Acadêmica do Curso de Terapia Ocupacional da Universidade Federal do Paraná. Laboratório de Reabilitação, Acessibilidade e Trabalho (LABRAT).

5. Mestranda do Programa de Pós-graduação em Administração - Ênfase em Estado e Trabalho da Universidade Federal da Paraíba, Laboratório de Saúde, Trabalho e Ergonomia (LASTE).

6. Professora Adjunta do Departamento de Terapia Ocupacional da Universidade Federal da Paraíba, Laboratório de Saúde, Trabalho e Ergonomia (LASTE).

Endereço para correspondência: Av. Prefeito Lothário Meissner, 3400. Prédio Didático II do Setor de Ciências da Saúde - Jardim Botânico, Curitiba, PR. CEP: 80210-170. E-mail: iranise@ufpr.br 


\section{INTRODUÇÃO}

Terapia Ocupacional é definida como o uso
terapêutico de ocupações, com objetivo de
melhorar ou possibilitar a participação em papéis, hábitos e rotinas em diversos ambientes (como casa, escola, local de trabalho, comunidade e outros). Os serviços de Terapia Ocupacional visam à habilitação, reabilitação e promoção da saúde e do bem-estar em sujeitos com necessidades relacionadas ou não a incapacidade. Tais serviços incluem a aquisição e preservação da identidade ocupacional, prevenção e reabilitação de enfermidades, lesões, doenças, desordens, problemas, deficiências, incapacidades, limitações de atividade ou restrições na participação ${ }^{1}$.

No campo profissional, a Terapia Ocupacional tem se firmado desde a regulamentação da profissão em 1969 e mais expressivamente a partir da década de 1980 com a institucionalização do currículo mínimo para a graduação e no século XXI com as diretrizes curriculares ${ }^{2}$.

No Brasil, a formação em Terapia Ocupacional desenvolveu-se por intermédio de influências internacionais, devido à demanda de profissionais na atenção asilar aos doentes mentais, e na reabilitação física, com o propósito de, através de atividades recreativas ocupacionais e laborterápicas e da restauração da capacidade funcional e do desempenho ocupacional na vida cotidiana, respectivamente, tornar novamente funcionais os indivíduos que possuíssem alguma limitação e/ou incapacidade, de modo que os primeiros cursos instituídos no Brasil eram especificamente voltados para estas vertentes ${ }^{3}$.

Para ampliar a abrangência e o reconhecimento da profissão, é fundamental que haja consolidação da formação na graduação e pós-graduação (stricto $e$ lato sensu), nas distintas áreas de atuação, buscando respaldar as áreas de construção de conhecimento em terapia ocupacional. Dentre diversas áreas de atuação, destacamos a intervenção em Saúde do Trabalhador, que inclui o campo de saberes e práticas inerentes à tríade "saúde, doença e trabalho", na qual o terapeuta atua em prevenção, promoção e reabilitação, quanto à vigilância, assistência, reabilitação física, funcional e profissional em saúde do trabalhador e inclusão de pessoas com deficiência no trabalho, possibilitando, assim, benefícios à qualidade de vida do trabalhador.

No Brasil, a reabilitação profissional de indivíduos incapacitados para o trabalho se configurou como uma das principais vertentes da construção da Terapia Ocupacional, juntamente com as práticas asilares destinadas às pessoas com transtornos mentais ${ }^{4}$.
De acordo com Moreira ${ }^{3}$, a formação em Terapia Ocupacional, inicialmene tecnicista e centralizada nas instituições reabilitadoras por meio de estratégias de reabilitação funcionais ou profissionais, passou a ser revista a partir do questionamento crítico acerca de sua prática, o que permitiu a reformulação de suas ações.

Entende-se a construção da Saúde do Trabalhador no Brasil como um processo sócio-histórico, iniciado na década de 1970, resultante de lutas e movimentos sociais marcados pelo processo de redemocratização e reformas na área da saúde brasileira ${ }^{5}$.

As preocupações com a saúde dos trabalhadores transformaram-se ao longo das décadas, acompanhando as mudanças sociais, políticas, econômicas, organizacionais e os avanços tecnológicos ${ }^{6}$.

Desde então, este segmento consolida-se a partir de diversas portarias e decretos, regulamentados pelo Ministério da Saúde. Destacam-se, nesse âmbito, a lista de Doenças Relacionadas ao Trabalho, que oferece subsídios para orientar os profissionais da saúde no que se refere aos agravos gerados no processo de trabalho, incluindo a Política Nacional de Saúde e Segurança do Trabalhador $(\mathrm{PNSST})^{7}$.

Soma-se a esse quadro a criação da Rede Nacional de Atenção Integral em Saúde do Trabalhador (RENAST) ${ }^{5}$ que evidencia a institucionalização da Saúde do Trabalhador em ações na rede de Atenção Básica, nos Centros de Referências em Saúde do Trabalhador (CEREST) e na rede assistencial de média e alta complexidade do SUS.

Compreende-se que tanto os acidentes, quanto as doenças associadas ao trabalho podem ser evitadas, caso haja planejamento e intervenção específica. Os múltiplos aspectos desse contexto (físicos, psíquicos, emocionais e sociais) constroem a perspectiva voltada para ações e articulações multiprofissionais, interdisciplinares e intersetoriais. Neste contexto, a Terapia Ocupacional pode contribuir nos contextos de trabalho, considerando o ambiente, as relações e os processos de trabalho, nos procedimentos de reabilitação individual, e no retorno e permanecia no trabalho, aspirando à atenção integral dos trabalhadores assistidos ${ }^{5}$.

Estudar as situações de trabalhado levando em consideração à atenção integral implica em vigilância e assistência em saúde do trabalhador; prevenção de agravos e promoção da saúde; reabilitação e retorno ao trabalho, de modo a conhecer as demandas dos trabalhadores e desenvolver ações específicas de prevenção, promoção, educação em saúde, proteção e reabilitação conforme propostas da Relação Nacional de Ações e Serviços de Saúde (RENAES) ${ }^{5}$. 
A formação em Terapia Ocupacional decorre de inúmeras reformulações provocadas pelo contexto histórico e político de cada época, de forma a atender a formação de profissionais críticos e qualificados, cabendo constante discussão e avaliação acerca da formação ofertada9 .

$\mathrm{O}$ desenvolvimento do ensino articulado à pesquisa e extensão de serviços à comunidade, bem como a capacitação docente têm importância fundamental diante do crescimento dos cursos de graduação, principalmente a partir de 1998. Tal expansão recoloca desafios para docentes e profissionais no que diz respeito à sua estruturação e consolidação ${ }^{10}$.

De acordo com Emmel e Lancman ${ }^{11}$, no Brasil, um grupo de docentes vem trabalhando desde 1986 sobre otema. A Rede Nacional de Ensino e Pesquisa em Terapia Ocupacional (RENETO), vem realizando encontros nacionais a fim de promover e fomentar a discussão, entre docentes e pesquisadores, sobre a formação profissional e a produção de conhecimento. Dessa maneira, as universidades detêm papel relevante para a configuração e fomento dos campos de atuação por meio da produção de conhecimentos específicos, promoção de debates relevantes, difusão de ideias inovadoras e formação de profissionais capacitados.

\section{OBJETIVOS}

Esta pesquisa possui como objetivo geral a investigação do desenvolvimento da área de conhecimento da Saúde do Trabalhador nos cursos de graduação em Terapia Ocupacional das instituições públicas de ensino superior (federais e estaduais) brasileiras. Como objetivos específicos: verificar se os cursos de graduação contemplam a área de Saúde do Trabalhador, elencaras disciplinas e estágios profissionais curriculares oferecidos; averiguar se os cursos possuem docentes capacitados na área de Saúde e Trabalho, e qual sua formação (especialização, mestrado, doutorado ou pós-doutorado), bem como quantificálos; examinar se os cursos desenvolvem pesquisas, iniciações científicas e projetos de extensão no contexto do trabalho e quais as temáticas mais pesquisadas; além de conhecer e descrever a estrutura dessas instituições relativa a laboratórios e grupos de pesquisas cujas linhas de investigação contemplem a Saúde do Trabalhador.

\section{PROCEDIMENTOS METODOLÓGICOS}

A pesquisa é resultado de projeto de iniciação científica vinculado ao Laboratório de Reabilitação, Acessibilidade e Trabalho (LABRAT), do Departamento de Terapia Ocupacional, da Universidade Federal do Paraná (UFPR) em consórcio com o Laboratório de Saúde, Trabalho e Ergonomia (LASTE) da Universidade Federal da Paraíba (UFPB).

Trata-se de um estudo que combina abordagens quantitativa e qualitativa, do tipo descritivo-exploratório, que utilizou, como meios para responder suas indagações, a pesquisa documental com a aplicação de questionários, através da ferramenta Google Drive.

Tem-se que, face ao objetivo proposto, a abordagem quantitativa permitirá definir quantitativamente o cenário do ensino de Saúde do Trabalhador nos cursos de graduação em Terapia Ocupacional nas instituições de ensino superior no Brasil, os quais receberão tratamento estatístico descritivo, por meio de tabulação simples dos dados, permitindo demonstrar e apresentar quais e o que os cursos de Terapia Ocupacional oferecem no âmbito do ensino da área de Saúde do Trabalhador.

Optou-se, ainda, pela abordagem qualitativa, pois está modalidade objetiva apreender a percepção, compreensão e participação dos sujeitos sobre o problema investigado e os aspectos relacionais existentes no fenômeno estudado ${ }^{12}$, que neste estudo corresponde ao conjunto de fatores organizacionais e estruturais que determinam a ênfase dada a uma determinada área de conhecimento no ensino da Terapia Ocupacional e suas influências na formação do graduando.

\section{Amostra}

As instituições foram selecionadas a partir dos seguintes critérios de inclusão: ser integrante de uma instituição de ensino superior pública (federal ou estadual); oferecer o curso de graduação em Terapia Ocupacional.

Os dados foram coletados de abril a setembro de 2015, em amostra não probabilística por conveniência, composta pelas instituições que aceitaram participar da pesquisa e responderam o questionário, portanto, contemplada por $(\mathrm{n}=16)$ cursos de instituições públicas de ensino superior brasileiras, dentre o universo de $(n=22)$ cursos de graduação em Terapia Ocupacional identificados.

\section{Instrumento da coleta de dados}

O instrumento utilizado foi um questionário semiestruturado com questões abertas, que abordou aspectos sobre disciplinas, estágios profissionais e projetos de pesquisa e de extensão ofertados, tanto no projeto pedagógico como na grade curricular dos cursos a respeito do ensino em Saúde do Trabalhador. 


\section{Procedimentos para a coleta e análise de dados}

Inicialmente realizou-se um levantamento de fontes bibliográficas e base de dados acerca do tema a ser discutido, utilizando-se de artigos e livros com o objetivo de estabelecer a discussão a respeito do ensino de Saúde do Trabalhador nos cursos de graduação em Terapia Ocupacional, na perspectiva da realidade brasileira. Após análise e compreensão da problemática levantada, a pesquisa foi submetida à avaliação e aprovação pelo Comitê de Ética em Pesquisa (CEP), do Setor de Ciências da Saúde da UFPR em 01/10/2014, tendo obtido Certificado de Apresentação para Apreciação Ética (CAAE) de número 276714.8.0000.0102. Após aprovação, foi realizado contato via telefone e e-mail institucional com as coordenações dos cursos de graduação, a fim de atualizar os dados para contato.

Dando seguimento, foi encaminhado via e-mail a carta-convite e o aceite institucional. Após recebimento do aceite institucional devidamente assinado, foi enviado o Termo de Consentimento Livre e Esclarecido (TCLE) de acordo com a Resolução 466/2012 do Conselho Nacional de Saúde. Após serem realizados os trâmites relacionados à documentação, os participantes tiveram acesso à pesquisa, via questionário.

Os dados coletados receberam tratamento estatístico descritivo, por meio de tabulação simples, sistematizada a fim de apresentar a realidade do ensino de Saúde do Trabalhador nos cursos de graduação em Terapia Ocupacional nas instituições públicas de ensino superior do Brasil. Conforme os preceitos éticos descritos na resolução supracitada, bem como, no TCLE, mantémse o sigilo pessoal da amostra desta pesquisa.

\section{RESULTADOS}

As instituições de educação e cursos cadastrados até $2015^{13}$ mostram a existência de 72 cursos no país ${ }^{13}$, dos quais cinco foram extintos e seis estão em processo de extinção (todos provenientes de universidades privadas). A amostra foi caracterizada a priori por $(\mathrm{n}=22)$ universidades públicas representando $30,5 \%$ dos cursos ofertados no país, com distribuição desigual entre as regiões brasileiras, conforme o Quadro 1.

A UFPR oferta o curso nos períodos diurno e noturno - é necessário esclarecer que os dois turnos disponibilizados, apesar de possuírem o mesmo plano pedagógico e grade curricular, são contabilizados nesta pesquisa como dois cursos diferentes, estabelecidos na mesma universidade.
Quadro 1 - Instituições públicas de ensino superior que ofertam curso de Terapia Ocupacional no Brasil

\begin{tabular}{|l|l|l|}
\hline Região & Estado & IES Públicas \\
\hline $\begin{array}{l}\text { Centro- } \\
\text {-Oeste }\end{array}$ & $\begin{array}{l}\text { Distrito } \\
\text { Federal }\end{array}$ & Universidade de Brasília (UnB) \\
\hline \multirow{5}{*}{ Nordeste } & Alagoas & $\begin{array}{l}\text { Universidade Estadual de Alagoas } \\
\text { (UNCISAL) }\end{array}$ \\
\cline { 2 - 3 } & Pernambuco & $\begin{array}{l}\text { Pniversidade Federal da Paraíba } \\
\text { (UFPB) }\end{array}$ \\
\cline { 2 - 3 } Pernambuco (UFPE)
\end{tabular}

Durante o período de coleta de dados, foram recebidos 17 questionários, sendo que, em uma das universidades, apesar de ter o curso aprovado e constar no cadastro do Ministério da Educação (MEC), ainda não existe formação de turma, configurando, portanto, uma amostra de 16 cursos, em 15 universidades, o que representa $73 \%$ dos cursos ofertados em instituições públicas de ensino superior no país. 
Quadro 2 - Conteúdos programáticos

\begin{tabular}{|c|c|}
\hline Disciplina específica & Disciplinas correlatas \\
\hline Conteúdo programático & Conteúdo programático \\
\hline \multirow{4}{*}{$\begin{array}{l}\text { Conceitos de saúde e } \\
\text { trabalho; Aspectos sócio- } \\
\text { históricos da saúde do } \\
\text { trabalhador; Legislação; } \\
\text { Políticas públicas de saúde } \\
\text { e segurança no trabalho; } \\
\text { Acidentes de trabalho, } \\
\text { doenças ocupacionais, } \\
\text { saúde mental e qualidade } \\
\text { de vida no trabalho; } \\
\text { Psicodinâmica do trabalho; } \\
\text { Ergonomia; Inclusão e } \\
\text { Reabilitação profissional; } \\
\text { Interface saúde, trabalho } \\
\text { e Terapia Ocupacional; } \\
\text { Avaliações e intervenções } \\
\text { da Terapia Ocupacional em } \\
\text { Saúde do Trabalhador, nos } \\
\text { diversos âmbitos da atenção } \\
\text { à saúde. }\end{array}$} & Ergonomia \\
\hline & $\begin{array}{l}\text { Conceitos ergonômicos } \\
\text { e ergológicos, aspectos } \\
\text { históricos e conceituais; } \\
\text { Considerações fisiológicas, } \\
\text { biomecânicas e } \\
\text { antropométricas voltada para } \\
\text { a saúde ocupacional; Análise } \\
\text { Pluridisciplinar das Situações } \\
\text { de Trabalho (APST); Normas } \\
\text { Brasileiras em Saúde do } \\
\text { Trabalhador e o processo de } \\
\text { intervenção ergonômica. }\end{array}$ \\
\hline & Saúde do adulto \\
\hline & $\begin{array}{l}\text { Psicodinâmica do trabalho; } \\
\text { Aspectos sócio-históricos } \\
\text { da Saúde do Trabalhador no } \\
\text { Brasil; Legislação; ações } \\
\text { da Terapia Ocupacional; } \\
\text { Ergonomia. }\end{array}$ \\
\hline
\end{tabular}

Destas, apenas duas instituições não oferecem disciplina específica de Saúde do Trabalhador, porém, apresentam uma disciplina correlata, a exemplo da Ergonomia, geralmente caracterizada em eixo transversal, como na Saúde do Adulto. As disciplinas ofertadas possuem carga horária de 60 a 80 horas, abordam aspectos sóciohistóricos da saúde do trabalhador, da relação trabalhosaúde-doença e sua interface com a Terapia Ocupacional, legislação, políticas públicas, psicodinâmica do trabalho, ergonomia, e intervenções da Terapia Ocupacional em Saúde do Trabalhador, nos diversos âmbitos da atenção à saúde (Quadro 2). Dentre todas as universidades participantes da pesquisa apenas uma possui carga horária prática específica (30 horas); as demais dispõem de atividades práticas, como visitas técnicas, palestras de convidados e estudos de caso.

Além das disciplinas específicas, cinco cursos apresentaram, disciplinas correlatas, como ergonomia e abordagem em eixos da saúde coletiva, tecnologia assistiva e habilidades profissionais em Terapia Ocupacional, nas quais são discutidas questões de ergonomia (conceitos, análise e aplicabilidade), adaptações para o trabalho, políticas públicas e práticas profissionais.

Dez instituições participantes possuem docentes com titulação na área de concentração de Saúde e Trabalho ou em áreas correlatas, sendo um especialista em Saúde do Trabalhador; quatro mestres, dos quais três na área de Ergonomia e um na de Psicodinâmica do Trabalho; cinco doutores, dos quais três na área de Ergonomia, um na de
Ciência da Reabilitação e um na área de Educação, que não foi aqui considerada como área correlata; e, apenas um docente com pós-doutorado, na área de Psicodinâmica do Trabalho. A Tabela 1 apresenta um panorama acerca de projetos, pesquisas e produção com relação à qualificação dos discentes responsáveis pelas disciplinas.

Nota-se que as instituições em que os professores possuem qualificação específica apresentam maior produção teórica, projetos de pesquisa e extensão, além de ofertarem estágio na área.

De acordo com os dados coletados, 50\% das instituições $(n=7,5)$ oferecem estágios na área de Saúde do Trabalhador, compreendendo estágios de prática direta em ergonomia, promoção à saúde do trabalhador, reabilitação (acidentes de trabalho e doenças ocupacionais) e inclusão profissional, a partir do $4^{\circ}$ período. Ainda, uma das universidades apresenta possibilidade de realização de estágio extracurricular, no âmbito da ergonomia, pois possui um Laboratório de Pesquisa na área de Saúde, Trabalho e Ergonomia, com diversos programas de pesquisa e extensão, bem como, possui parcerias com o outros Departamentos na mesma Universidade (Engenharia de Produção e Fisioterapia).

Apenas nove instituições possuem projetos de extensão na área de Saúde do Trabalhador, dispostas nos seguintes âmbitos: ergonomia, condições de trabalho de migrantes da construção civil, sistema prisional e inclusão profissional, totalizando-se 14 ações na área.

Quanto à produção de trabalhos de conclusão de curso, nos últimos três anos, observou-se que todas as instituições apresentaram produção na área de saúde e trabalho, contabilizando em média 4,3 trabalhos por entidade. Identificou-se também que existiu um total de 36 pesquisas, uma média de 2,2 pesquisas por instituição, observando-se diferenças mínimas na comparação entre as que tinham professores pós-graduados e aquelas que dispunham apenas de docentes com graduação, sendo 2,8 e 2,5 pesquisas por instituição, respectivamente.

Com relação às publicações, nota-se maior percentual nas universidades com professores pósgraduados $(n=10)$, com média de 2,8 publicações por instituição, enquanto que, nas demais, houve apenas uma publicação. De forma geral, as 29 publicações são resultantes de trabalhos de conclusão de curso e pesquisas relacionadas a ergonomia, psicodinâmica do trabalho, acidentes de trabalho/doenças ocupacionais, tecnologia assistiva, indicadores de saúde em trabalhadores, tradução e adaptação transcultural de instrumentos, e, reabilitação e readaptação funcional. Do total de publicações, 9 foram em revistas internacionais, 13 em periódicos de Terapia Ocupacional e sete em revistas de áreas correlatas.

Considerando o período de análise, tem-se em média 23 trabalhos de conclusão de curso e 9,6 pesquisas realizadas por ano na área de saúde e trabalho. Com 
relação às publicações referente ao tema, o ano de maior número de artigos publicados foi em 2015, com um total de 9 publicações.

Tabela 1 - Panorama geral do ensino de Saúde do Trabalhador nos cursos de Terapia Ocupacional nas instituições públicas de ensino superior no Brasil

\begin{tabular}{l|c|c|c}
\hline & $\begin{array}{c}\text { Com } \\
\text { professores } \\
\text { titulados }\end{array}$ & $\begin{array}{c}\text { Sem } \\
\text { professores } \\
\text { titulados }\end{array}$ & Total \\
\hline $\begin{array}{l}\text { Instituições de } \\
\text { ensino superior }\end{array}$ & 10 & 06 & 16 \\
\hline Estágios ofertados & 08 & 08 & 16 \\
\hline $\begin{array}{l}\text { Programas de } \\
\text { extensão }\end{array}$ & 10 & 06 & 16 \\
\hline $\begin{array}{l}\text { Trabalho de } \\
\text { conclusão de curso }\end{array}$ & 63 & 07 & 70 \\
\hline Pesquisa & 14 & 06 & 20 \\
\hline $\begin{array}{l}\text { Publicação em } \\
\text { periódicos }\end{array}$ & 28 & 01 & 29 \\
\hline
\end{tabular}

\section{DISCUSSÃO}

Segundo Oliver ${ }^{3}$, em 2004, havia, no país, 39 cursos de Terapia Ocupacional, 9 deles em universidades públicas e 30 em universidades privadas ${ }^{8}$, e em julho de 2008, dados do Instituto Nacional de Estudos e Pesquisas Educacionais Anísio Teixeira ${ }^{14}$ identificavam 63 cursos de graduação, a maior parte nos estados de São Paulo e Minas Gerais. Neste período, 12 cursos se desenvolviam em universidades públicas, instituições onde têm sido mais favoráveis as condições para a elaboração de pesquisas articuladas à graduação e à extensão de serviços à comunidade. Em instituições privadas, foram identificados, nesse período, 51 cursos.

Em 2014, existiam 72 cursos credenciados pelo MEC no país, sendo 22 em universidades públicas, estaduais e federais, e 50 em universidades privadas ${ }^{13}$. Verificou-se, portanto, que entre 2004 e 2014 foi notável a expansão de $84,61 \%$ do referido curso nas instituições de ensino superior, apresentando um aumento de $144,44 \%$ nas instituições públicas. Ressalta-se que este número referese aos cursos credenciados e não necessariamente em funcionamento. Estima-se que 50 cursos dos cadastrados estão em funcionamento atualmente.

Foi verificado que todos os cursos analisados, abordam a área de Saúde do Trabalhador. Em sua maioria dispõem de professores com pós-graduação na área de Ergonomia. Os resultados apresentados nos mostram que, de modo geral, a temática da área de Trabalho estão presentes em todos os cursos das diferentes instituições públicas. No entanto, isto se dá de forma diferenciada em cada uma das instituições que responderam a pesquisa, no que tange os seguintes critérios: quantidade de disciplinas ofertadas pelos cursos; ementa curricular; estágios; trabalhos de conclusão de curso e publicações em formato de artigo; programas de pesquisa e extensão; quantidade de recursos humanos destinados especificamente à área $\mathrm{e}$ titulação; e, perfil do docente.

Assim, pode-se observar que as instituições públicas de ensino superior que possuem professores com qualificação específica na área de Saúde do Trabalhador apresentam maior número de estágios, trabalhos de conclusão de curso, projetos de extensão, projetos de pesquisa e publicações nesse campo. Porém, as titulações de pós-graduação dos docentes concentram-se apenas em uma área, recomendando-se, assim, a ampliação de temas na formação dos discentes e ampliação de linhas de pesquisa na formação acadêmica.

A falta de recursos humanos com formação mais abrangente, fora a área de Ergonomia, associada ao número de docentes na área de Trabalho foi um problema encontrado; isto está relacionado diretamente com as dificuldades relativas a formulação de programas de pesquisa e extensão desde a graduação, passando pelos programas de pós-graduações na área.

A análise das emendas das disciplinas, apresentaram a distribuição dos conteúdos ministrados ao longo da graduação, os quais em sua maioria abrangem aspectos sóciohistóricos da Saúde do Trabalhador, incluindo a evolução da legislação e políticas públicas, a relação trabalho-saúdedoença e sua interface com a Terapia Ocupacional, através da análise da psicodinâmica do trabalho e da ergonomia.

Além disso, inserem-se as intervenções da Terapia Ocupacional em Saúde do Trabalhador nos diversos âmbitos da atenção à saúde, de modo que é exigida maior compreensão acerca da construção social dos conceitos de trabalho versus doença, dos processos de Terapia Ocupacional e de ferramentas de avaliação e técnicas de aplicação de intervenções terapêuticas, justificando, portanto, sua ministração entre o $5^{\circ}$ e o $8^{\circ}$ período. Tal atitude permite que os acadêmicos desfrutem da disciplina de forma mais completa, visto o somatório de conhecimentos e experiências adquiridos.

Como forma de ampliar a formação discente na área de Trabalho, se faz necessário que os projetos dos cursos das instituições públicas, contemplem explicitamente a área de Trabalho em suas matrizes curriculares, desde as disciplinas básicas até os estágios.

Apesar do crescimento da oferta de opções ligadas à formação em Terapia Ocupacional, Lopes e outros ${ }^{15}$ apontam o decréscimo do número de alunos e a falta de políticas assistenciais relacionadas à oferta de trabalho como principais desafios quanto à manutenção dessas instituições, fato que indica a necessidade de novas discussões voltadas à temática central desta pesquisa, com vistas à formação de profissionais qualificados e comprometidos, que sejam ativos e compartilhem de uma reflexão crítica. 


\section{CONSIDERAÇÕES FINAIS}

Embora tenham sido implementadas novas políticas e serviços em Saúde do Trabalhador que incluem a atuação do terapeuta ocupacional, a formação destes profissionais merece maior atenção quanto a suas especificidades, devendo permitir que se apropriarem de diversos métodos, técnicas, pressupostos teóricos e conceitos específicos do campo, de modo a sistematizar e aumentar a qualidade de seu trabalho, atendendo às conjecturas das ações e metodologias previstas nos serviços.

Os resultados deste estudo delinearam a produção de conhecimento sobre a formação de Terapia Ocupacional no

\section{REFERÊNCIAS}

1. American Occupational Therapy Association, A. Estrutura da prática da Terapia Ocupacional: domínio \& processo - 3a ed. trad. Rev Ter Ocup Univ São Paulo. 2015;26(esp):1-49. DOI: http://dx.doi.org/10.11606/issn.2238-6149.v26iespp1-49.

2. Oliver FC. Pesquisa e produção bibliográfica em Terapia Ocupacional: contribuições para o debate sobre parâmetros de avaliação da produção acadêmica brasileira. Rev Ter Ocup Univ São Paulo. 2008;19(2):108-20. DOI: http:// dx.doi.org/10.11606/issn.2238-6149.v19i2p108-120.

3. Moreira AB. Terapia Ocupacional: história crítica e abordagens territoriais/comunitárias. Rev Vita et Sanitas. 2008;2(2):79-91. Disponível em: www.fugedu.com.br/ novarevista/index.php/vitaetsanitas/issue/download/25/64.

4. Lancman S, Ghirardi MIG. Introdução. In: Lancman S, organizadora. Saúde, trabalho e terapia ocupacional. São Paulo: Roca; 2004. p.9-13.

5. Simonelli AP, Rodrigues DS, Lima J. A atuação da terapia ocupacional na saúde do trabalhador. In: Simonelli AP, Rodrigues DS, organizadoras. Saúde e trabalho em debate: velhas questões, novas perspectivas. Brasília: Paralelo 15; 2013. p.225-40.

6. Lancman S. Psicodinâmica do Trabalho. In: Cavalcanti A, Galvão C. Terapia ocupacional: fundamentação e prática. Rio de Janeiro: Guanabara Koogan; 2007. p.271-77.

7. Dias EC, Silva TL. Contribuições da atenção primária em saúde para a implementação da Política Nacional de Saúde e Segurança no Trabalho (PNSST). Rev Bras Saude Ocup. 2013;38(127):31-43. DOI: http://dx.doi.org/10.1590/S030376572013000100007.

8. Macedo AR et al. Educação superior no século XXI e a reforma universitária brasileira. Rev Ensaio Aval Políticas Públicas Educ. 2005;13(47):127-48. DOI: http://dx.doi. org/10.1590/S0104-40362005000200002.

Recebido em: 30.10 .16

Aceito em: 13.01 .16 âmbito da Saúde do Trabalhador nas instituições públicas de ensino superior do país. No entanto, identificou-se como barreira para a elaboração desta pesquisa a ausência de retorno, ou até mesmo o retorno tardio, por parte dos coordenadores dos cursos, o que dificultou a coleta dos dados em todas as universidades sujeitas a esta pesquisa.

Por fim, entende-se que a temática explorada na pesquisa é relevante para a formação e exercício do terapeuta ocupacional no âmbito da Saúde do Trabalhador, pois se verificou o evidente cenário de ampliação e alteração curricular nos cursos de graduação em Terapia Ocupacional no país, o que possibilita a expansão do acesso à formação do profissional nesta área, explicitando a importância de manter os padrões de qualidade em tal formação.

9. Tirado MGA, et al. Terapia ocupacional. In: Haddad AE, et al., organizadores. A trajetória dos cursos de graduação na saúde 1991-2004. Brasília: MEC; 2006. p.489-525.

10. Emmel MLG. Caracterização dos cursos de Terapia Ocupacional do Brasil: perspectivas frente à capacitação docente [mimeo]. São Carlos; 2004.

11. Emmel MLG, Lancman S. Quem são nossos mestres e doutores? $O$ avanço da capacitação docente em Terapia Ocupacional no Brasil. Cad Ter Ocup UFSCar. 1998;7(1):29-38. Disponível em: http://www. cadernosdeterapiaocupacional.ufscar.br/index.php/ cadernos/article/view/264/216.

12. Minayo MCS.O desafio do conhecimento. Pesquisa qualitativa em saúde. São Paulo: Hucitec; 2006.

13. Brasil. Ministério da Educação. Instituições de educação e cursos cadastrados. Brasília; 2015 [citado 24 set. 2015]. Disponível em: http://emec.mec.gov.br/.

14. Instituto de Pesquisas Educacionais Anísio Teixeira (INEP). Cursos de graduação 2008 [citado 30 jul. 2008]. Disponível em: http://www.educacaosuperior.inep.gov.br/funcional/ busca_curso.stm apud Moreira AB. Terapia Ocupacional: história crítica e abordagens territoriais/comunitárias. Rev Vita et Sanitas. 2008;2(2):79-91. Disponível em: www. fugedu.com.br/novarevista/index.php/vitaetsanitas/issue/ download/25/64.

15. Lopes RE, et al. XI Encontro Nacional de Docentes de Terapia Ocupacional: refletindo sobre os processos de formação acadêmica e profissional. Rev Ter Ocup Univ São Paulo. 2008;19(3):159-66. DOI: http://dx.doi.org/10.11606/ issn.2238-6149.v19i3p159-166. 